\title{
Gundelrebe (Glechoma hederacea) - Verursacher to̊dlicher Vergiftungen beim Pferd?
}

\author{
Eine Spurensuche
}

Renate Vanselow, Cäcilia Brendieck-Worm

\section{() Zusammenfassung \\ Glechoma hederacea steht unter Ver- dacht, beim Pferd tödliche Vergiftungen verursachen zu können. Das genaue Stu- dium der diesem Verdacht zugrunde lie- genden Literatur zeigt, dass bei diesen Vergiftungen besondere Bedingungen vorlagen: die alleinige Fütterung von hochgradig (>30\%) mit Gundelrebe ver- unreinigter Luzerne oder die experimen- telle, ausschließliche Fütterung mit Gun- delrebe.}

Gundelrebe (Glechoma hederacea), auch Gundermann genannt (\$ Kasten 1), wird seit Jahrzehnten in der Fachliteratur als toxisch für Tiere, insbesondere für Pferde, dargestellt $[4,7,9,14]$. Biologen und Veterinärmediziner werden seit einigen Jahren immer wieder von Tierhaltern, aber auch Redaktionen von Pferdezeitschriften und landwirtschaftlichen Wochenblättern um Stellungnahme zur Giftigkeit der Gundelrebe gebeten - ohne dass hierzu aktuelle Vergiftungsfälle Anlass gäben.

Für den Menschen gilt Gundelrebe als ungiftig, und sie wird in der Volksmedizin seit Jahrhunderten als heilkräftige Pflanze geschätzt. Es stellen sich also die Fragen: „Was ist dran an der Giftigkeit dieser Pflanze für Tiere, welche Wirkstoffe enthält sie, was ist bekannt über die Wirksamkeit und bei welchen Tieren traten Vergiftungen auf?“

\section{Traditionelle Nutzung in Europa}

Gundelrebe wurde von unseren Vorfahren umfangreich genutzt. So verwendeten u.a. die Sachsen Gundelrebe als Zutat zum Bier, bevor sich der Hopfen als Grundstoff durchsetzte. Diese Verwendung in der Brauerei schlägt sich noch heute in englischen Namen für die Gundelrebe nieder:

\section{Kasten 1: Steckbrief Gundelrebe}

syn. Gundermann (Glechoma hederacea), Familie der Lamiaceae (Lippenblütler)

Verbreitung, Vorkommen

In ganz Europa bis in mittlere Gebirgslagen; in unseren Breiten ein häufiger Bodenbedecker an Waldrändern, Zäunen, Hecken und Wegrändern, meist in geringer Menge im Unterwuchs oder im Halbschatten von Sträuchern und Bäumen.

\section{Beschreibung und Fortpflanzung} Kriechende, ganzjährig grüne Pflanze mit lang gestielten, rundlich-herzförmigen, grob gekerbten Blättern (\$ Abb. 1). Die Blütentriebe erheben sich und bilden in den Blattachseln 1-3 blauviolette Blüten (Blütezeit IV-VI). Nach dem Blühen wachsen die Blütentriebe als Kriechtriebe weiter. Gundelrebe kriecht mit bis zu 1,30 m langen Ausläufern über den Boden (engl. Ground-Ivy, Boden-Efeu). An den gegenständigen Blattansätzen bilden sich Wurzeln, aus denen neue Pflanzen entstehen. Auch unterirdisch bildet Gundelrebe lange Ausläufer. Abgetrennte Wurzeln schlagen neu aus [4].

\section{Inhaltsstoffe}

Ätherische Öle (maximal 0,03-0,06\%, v. a. Monoterpenketone, daneben Sesquiterpene), Glechomafuran, Glechomanolid, Rosmarinsäure (ca. 1,5\%), Kaffeesäure, Ferulasäure, Sinapinsäure, Flavonoide (Cymarosid, Cosmosysrin, Hyperosid, Isoquercitrin, Luteolin-7-diglucosid), Triterpencarbonsäuren, u. a., $\alpha$ - und $\beta$-Ursolsäure und Oleanolsäure, Hydroxyfettsäuren [9].

Verwechslungsmöglichkeiten Gundelrebe wird oft mit dem blau blühenden Kriechenden Günsel (Ajuga reptans, - Abb. 2), der Roten Taubnessel (Lamium purpureum, $\$$ Abb. 3 ) oder der ebenfalls rot blühenden Stängelumfassenden Taubnessel (Lamium amplexicaule) verwechselt.

Alehoof, Tunhoof und Gill-over-theGround. Gundelrebe wurde als Gewürz genutzt. Im Frühjahr, zur Blütezeit, wurden die Blätter gesammelt und als Gemüse gekocht. In geringeren Mengen wurden die Vitamin-C-haltigen Blätter auch roh im Salat verspeist. Zudem fand die Gundelrebe Verwendung in der Käsebereitung als pflanzliches Lab [17].

Gundelrebe war in der traditionellen europäischen Medizin als Heil- und Zauberpflanze bekannt. Sie wurde bei Magen-Darm-Katarrhen, Durchfall, Husten und Bronchialleiden verwendet. Die harntreibende Wirkung wurde bei Blasen- und Steinleiden geschätzt und zur Ausleitung und Entgiftung genutzt. Hildegard von Bingen empfahl Gundelrebe bei Kopf- und Ohrenschmerzen. In Italien wird die Gundelrebe bei Arthritis und Rheuma eingesetzt. Die TCM kennt sie bei Lungenentzündung und Nephritis $[9,17]$.

\section{Moderne Medizin und Forschung}

Zurzeit spielt Gundelrebe in der rationalen Phytotherapie keine Rolle, sie ist jedoch vielfach Gegenstand aktueller phytopharmakologischer Forschung. Es wurden einzelne Inhaltsstoffe der Gundelrebe untersucht und dabei unterschiedlichste Wirkungen festgestellt. Entzündungshemmende Eigenschaften wurden an Mäusen und Ratten nachgewiesen [2], ebenso positive Wirkungen gegen Geschwüre durch Öle aus Glechoma hederacea. Ursolsäure zeigte eine antivirale Wirkung gegen das Epstein-Barr-Virus [2]. Sesquiterpenlaktone aus Glechoma wurden an Zelllinien in ihrer Wirksamkeit gegen Krebs beim Menschen erforscht [10] u. v.m.

\section{Vergiftungsfälle}

Die gesamte Fachliteratur der letzten Jahrzehnte (s. o.) bezieht sich mit ihren Aussagen über die Giftigkeit von Gundelrebe auf 2 Veröffentlichungen von Vergiftungsfäl- 

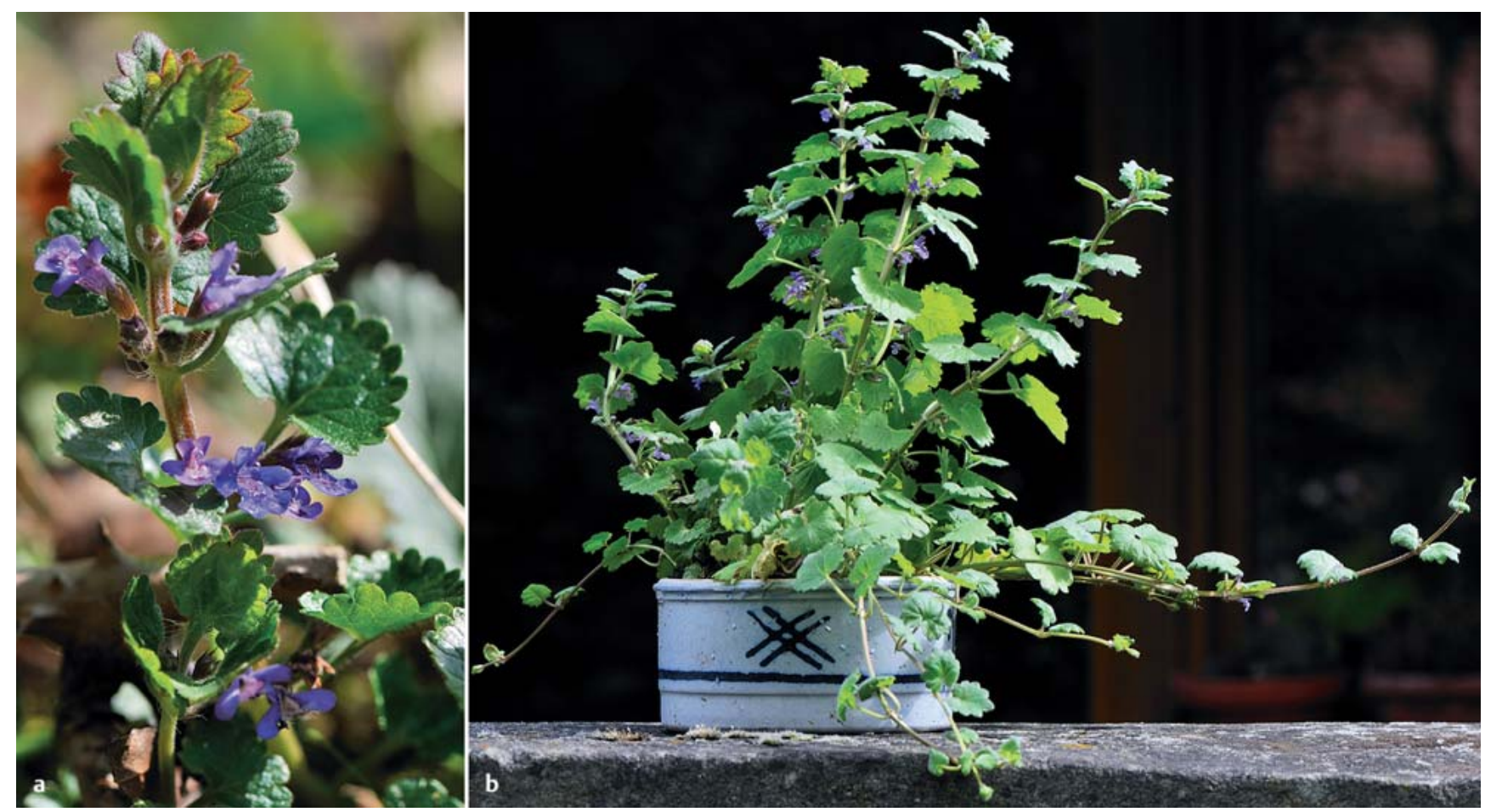

Abb. 1 a und b Gundelrebe (Glechoma hederacea): a im Freiland, b als „Zierpflanze“. @ Ferdinand Worm.

len bei Pferden aus den Jahren 1935 [8] und 1955 [12] aus Ungarn und Rumänien, die im Folgenden dargestellt werden. Nach dem derzeitigen Kenntnisstand der Autorinnen wurden darüber hinaus Vergiftungsfälle bei anderen Tieren bisher nicht beschrieben und neue Vergiftungsfälle bei Pferden seither nicht dokumentiert.

Formulierungen wie „Bei Tieren wurden Vergiftungen beobachtet, bei Pferden z.T. sogar tödliche“ [4] oder „Nur bei Tieren (besonders giftig bei Pferden) wurden Vergiftungserscheinungen beobachtet“ [14], sind daher als Verallgemeinerungen zu betrachten, die bisher einer sachlichen Grundlage entbehren.

\section{Vergiftungsfälle in Ungarn}

1935 berichtet Hazslinszky (veterinärmedizinische Sektion der Palatin-Josef-Universität, Ungarn) über Vergiftungen bei Pferden durch Glechoma hederacea [8].

Zwölf Arbeitspferde wurden ab Mitte Mai mit frisch gemähter Luzerne gefüttert, die einen großen Anteil Glechoma enthielt. Am 6. Tag dieser Fütterung erkrankten 7 Pferde plötzlich: Es zeigten sich Atemnot, beschleunigter, schwacher Puls, Blutungen in Lidbindehäuten, Fieber und Benommenheit.

Trotz sofortigen Futterwechsels und Therapie mit Kampfer, Arecolin (๑ Kasten
2) und Rhizinusöl genasen erst nach mehreren Tagen 6 der Pferde. Ein älteres Pferd verstarb nach 14 Tagen. Bei der Sektion fanden sich eine Vergrößerung der Milz, Erweiterung des Blinddarms und Gastroenteritis.

Hazslinszky konnte seinerseits lediglich auf die Veröffentlichung seines Landsmanns Ferenczhazy [8] zu vermutlichen Vergiftungen mit Gundelrebe zurückgreifen. Dieser berichtete 1914 von schweren Vergiftungserscheinungen ebenfalls nach der Verfütterung von mit Gundelrebe verunreinigter Luzerne. Differenzialdiagnostisch zog Hazslinszky aufgrund der Luzernefütterung eine sog. Kleekrankheit in Betracht ( $\$$ Kasten 3).

Zur Klärung der Ursache unternahm Hazslinszky einen Fütterungsversuch mit 3 gesunden Pferden, die nach 12-stündigem Nahrungsentzug folgende Futterrationen von eben der Fläche erhielten, durch deren Bewuchs es zu den oben beschriebenen Vergiftungserscheinungen gekommen war:

- Pferd Nr. 1: ausschließlich frische, etwas verwelkte, unkrautfreie Luzerne ad libitum*

* Bei Ad-libitum-Fütterung fressen Pferde bis zu $60 \mathrm{~kg}$ frisches Grün (Gras und Kräuter) proTag!
Kasten 2: Arecolin (Arecolinum hydrobromicum)

Arecolin, Alkaloid aus der Betelnuss (Arekanuss), ist ein Parasympathomimetikum.

Dosen von 0,01-0,05 g rufen bereits nach 5 Minuten beim Pferd Speichelfluss hervor. Außerdem wirkt Arecolin in Dosen von 0,05-0,1 g durch tetanische Kontraktion der Darmmuskulatur (Kolik!) und Anregung der Darmdrüsen abführend.

Es kommt zu Schweißausbruch, vermehrtem Nasenausfluss und Harndrang. Die Herztätigkeit wird verlangsamt. In großen Dosen bewirkt Arecolin Herzarrhythmien, starke Beschleunigung des Pulses und Senkung der Körpertemperatur.

Dosen von $0,25 \mathrm{~g}$ wirken für Pferde stark giftig, solche von $0,5 \mathrm{~g}$ tödlich durch Atemlähmung unter tetanischen Krämpfen [5].

Pferd Nr.2: ausschließlich frische, etwas verwelkte Gundelrebe ad libitum*

- Pferd Nr.3: 14 Tage altes Luzerneheu mit einem Anteil von $1 / 3-1 / 2$ an Gundelrebe ad libitum. Zusätzlich erhielten alle Pferde ausschließlich Wasser.

\section{Verlauf}

- Pferd Nr. 1 zeigte im Laufe des Versuchs keinerlei Krankheitssymptome.

- Pferd Nr. 2 weigerte sich am 1. Tag, das Futter aufzunehmen, es begann erst am 


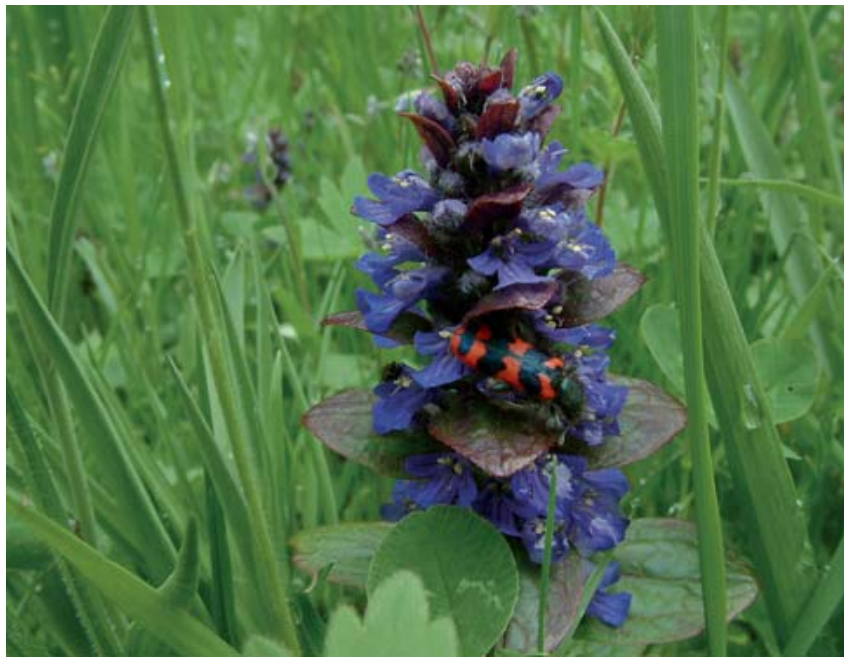

Abb. 2 Kriechender Günsel (Ajuga reptans). (c) Ferdinand Worm.

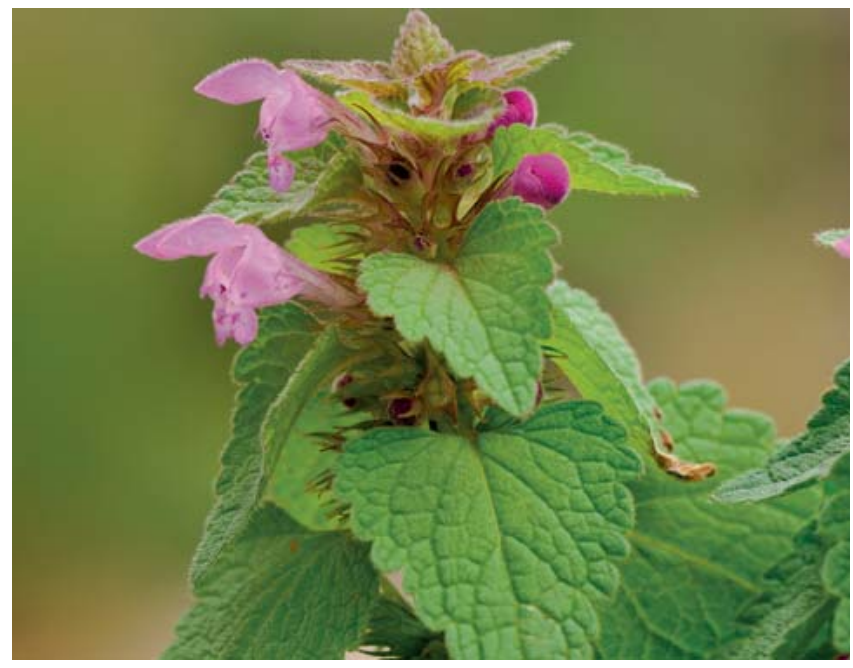

Abb. 3 Rote Taubnessel (Lamium purpureum). (c) Ferdinand Worm.

\section{Kasten 3: Giftige Inhaltsstoffe der Luzerne}

Luzerne (syn. Alfalfa, Medicago sativa) und Klee gehören zur Familie der Fabaceae, Tribus Trifolieae. Sie sind wertvolle, eiweißreiche Futterpflanzen, die in Symbiose mit sog. Knöllchenbakterien (Sinorhizobium meliloti) leben, die es ihnen ermöglichen, Stickstoff aus der Luft zu binden. Hierdurch tragen diese Pflanzen wesentlich zur Bodenverbesserung bei. Sie enthalten jedoch auch in Abhängigkeit von Jahreszeit und Witterung diverse Inhaltsstoffe, die bei einem Überangebot in der Futterration zu Schädigungen der Tiere führen können. Allgemein toxisch wirkende zyanogene Verbindungen inhibieren v. a. Enzymsysteme, die an Redoxprozessen beteiligt sind, blockieren z. B. die Atmungskette und führen zu Krämpfen. Diverse Isoflavone können als sog. Phytoöstrogene zu Fruchtbarkeitsstörungen führen [6].

Für Pferde gilt eine Tagesmenge von Klee- und Luzerneheu von 3-5 kg/500-600 kg KG bzw. $20 \mathrm{~kg}$ als frisches Grünfutter als maximale Futtermenge - in Kombination mit Futterstroh oder anderen rohfaserreichen, eiweißarmen Futtermitteln [13].

Nachmittag zu fressen. Am 4. Tag traten plötzlich Krankheitserscheinungen auf: Mattigkeit, geschwollene Adern und Blutungen in Augen und Lidbindehäuten, gelbliche Verfärbung der Maulschleimhäute, beschleunigter, kleiner, kaum fühlbarer Puls; beschleunigtes, röchelndes, nach Luft ringendes Atmen, wodurch der ganze Körper des Tieres ins Zittern kam; trotz bestehenden Durstes mühsames, schlürfendes Trin- ken; Verstopfung; Kolik; kalter Hals, Kopf und Ohren, Körpertemperatur $39-40{ }^{\circ} \mathrm{C}$, zunehmend benommen und reaktionslos.

- Die Gundermann-Fütterung wurde mit Auftreten der Symptome abgesetzt und das Tier mit Rizinusöl und Arecolin ( Kasten 2) behandelt. Unter Zunahme der Symptome starb das Tier am 10. Versuchstag. Bei der Sektion fanden sich eine Vergrößerung der Milz, Erweiterung des Blinddarms und Gastroenteritis.

- Pferd Nr. 3 erkrankte am 5. Versuchstag unter den gleichen Symptomen in milderer Ausprägung. Nach Absetzen der Fütterung und einer Injektion Arecolin war es am folgenden Tage wieder im Arbeitseinsatz!

Aus dem Verlauf dieses Versuchs schließt Hazslinszky, „dass die Gundelrebe eine nicht unbedeutende Giftpflanze ist, die frisch oder etwas verwelkt bei Pferden auch tödliche Vergiftungen verursachen kann und ihre giftige Wirkung noch einige Zeit - obgleich in geringerem Maße - auch in getrocknetem Zustande (im Heu) behält" [8].

Zur Klärung, wie lange diese vermutete Giftwirkung anhält, wurde ein 4. Pferd mit mehrere Monate altem Luzerneheu mit 60\%igem (!) Gundelrebeanteil 2 Wochen lang gefüttert. Es traten keine Krankheitssymptome auf, woraus geschlossen wurde, dass Gundelrebe seine Giftigkeit durch einige Monate Trocknung verliert.

\section{Diskussion}

Therapie mit Kampfer (innerliche Anwendung) und Arecolin gilt heute aufgrund der geringen therapeutischen Breite und des hohen Nebenwirkungspotenzials als obsolet [11]. Auch der Einsatz von pflanzlichen Ölen wie Rizinusöl als Laxanzien wird heute bei Vergiftungsverdacht abgelehnt, da sie die Resorption lipophiler Toxine begünstigen können [3].

Die von Hazslinszky eingesetzte Therapie muss demnach aus heutiger Sicht als kontraindiziert betrachtet werden. Die sicher bei Vergiftungsfällen indizierte Ausleitungstherapie kann mit wesentlich weniger drastischen Methoden durchgeführt werden (\$ Kasten 4).

Die Angabe, dass es sich bei dem verendeten Pferd aus der Gruppe der 7 erkrankten Tiere um ein älteres Tier handelte, lässt den Verdacht aufkommen, dass hier die Therapie eher zum schlechten Verlauf beigetragen haben könnte, denn der alte Organismus hat häufig bereits insuffiziente Entgiftungskapazitäten, wodurch unerwünschte Nebenwirkungen der Medikation eher auftreten können.

Das von Hazslinszky gewählte Versuchsdesign muss ebenfalls als unangemessen drastisch bezeichnet werden, da hier jede Dosis-Wirkungs-Beziehung außer Acht gelassen wurde.

Eine Futterration, die zu 100\% aus der vermutlich giftigen Pflanze bestand und die vom Versuchstier nur aufgenommen wurde, weil kein anderes Lebensmittel zur Verfügung stand, kann über den Grad der Giftigkeit dieser Pflanze wenig aussa- 
gen, fest steht lediglich, dass Gundelrebe als „ziemlich zahlreich“ (Hazslinszky) in Luzerne vorkommendes Unkraut zu Vergiftungen führen kann und als Alleinfuttermittel zur tödlichen Vergiftung eines Pferdes geführt hat.

Auch die Schlussfolgerung Hazslinszkys bezüglich der Giftigkeit im Heu ist infrage zu stellen. Frisches Heu kann auch ohne Gehalt an Gundelrebe die beschriebenen Symptome erzeugen ( $\$$ Kasten 5).

\section{Vergiftungsfälle in Rumänien}

Erst 20 Jahre später wurde von Nicolau et al. [12] erneut über Vergiftungsfälle bei Pferden berichtet, die man auf die Fütterung mit frisch gemähter Luzerne zurückführte, die einen großen Anteil Gundelrebe (32\% bzw. $48 \%$ der Ration) enthielt. Innerhalb weniger Tage erkrankten Ende Mai bis Anfang Juni 1955 in der Umgebung von Arad und Temeswar in Rumänien 42 Pferde, von denen 6 verendeten.

Die Untersuchung der Tiere zeigte eine Polypnoe mit bis zu 100 Atemzügen/Mi-
Kasten 4: Vorgehen zur

\section{Dekontamination}

wiederholte Magenspülung per NSS mit 4-5I frischem Wasser

Darmentleerung durch Laxanzien (Glaubersalz, 0,5-1 g/kg KGW als wässrige Lösung per NSS)

- Gifte binden durch Aktivkohle (Carbo medicinalis) $1-3 \mathrm{~g} / \mathrm{kg} \mathrm{KGW}$ als 20-30\%ige, wässrige Suspension per NSS, Wiederholung alle 8 Stunden, um die Rückresorption über den enterohepatischen Kreislauf zu unterbinden

renale Ausscheidung forcieren durch Infusion, ggf. mit Mannitol und Furosemid [3]

nute, ein alveoläres Lungenemphysem mit z.T. erhöhter Körpertemperatur, beschleunigten Puls mit geringer Spannung; Herzstoß und Darmgeräusche waren verstärkt und der Mastdarm erweitert. Die sichtbaren Schleimhäute aller Tiere waren gerötet, bei einigen konnten auch Schwellung und subikterische Verfärbung festgestellt werden. Im Blut zeigte sich eine Lym-

\section{Kasten 5: Frisches Heu}

Heu darf während der ersten 8-12 Wochen grundsätzlich nicht verfüttert werden. Während dieser sog. „Schwitzphase“ werden u. a. die Toxine der Hahnenfußgewächse abgebaut. Die Fütterung von frischem Heu verursacht u. U. Magen- und Darmkatarrhe und Hufrehe [1].

phozytose. Die Symptome traten 5 Tage nach Beginn der Luzernefütterung auf. Bei der Autopsie von 2 verendeten Pferden wurden eine Herzmuskeldegeneration, Hyperämie, ein Lungenödem und eine Stauungsleber festgestellt. Dieses Krankheitsbild war bis dato in Rumänien nicht beschrieben worden. Eine infektiöse Ursache wurde ausgeschlossen.

Die nach Ansicht von Nicolau et al. ursächliche Diagnose wurde in der Gemeinde Rovine-Pecica gestellt: Die Anamnese ergab, dass im 1. Betrieb neben der frischen Luzerne auch Gerecheltes, d. h. nach dem Absammeln der Luzerne abgerechtes 
und in der Hauptsache Gundelrebe enthaltendes Grünfutter verfüttert worden war.

Die Pferde erhielten erst wieder Futter, als die Krippen geleert waren. Von 11 Pferden erkrankten 8.

Im 2. Betrieb wurde kein Gerecheltes zur mit Gundelrebe durchsetzten Luzerne verfüttert. Von 10 Pferden erkrankten 4.

Im 3. und 4. Betrieb entfernten die Wärter das von den Pferden in der Krippe zurückgelassene Futter, das fast ausschließlich aus Gundelrebe bestand. Hier erkrankte kein Pferd [12].

Nicolau et al. fassen zusammen:

- Frisch gemähte Luzerne, die einen großen Prozentsatz Glechoma hederacea enthielt, konnte schwere Vergiftungen bei Pferden erzeugen.

- Das klinische Bild der Vergiftung war das eines akuten alveolären Lungenemphysems mit übertriebener Schnellatmigkeit und normaler oder erhöhter Temperatur.

Es lag eine Lymphozytose vor.

- In einem Drittel der Fälle gab es Komplikationen, die Mortalität lag bei 15\%.

- Sofortiger Futterwechsel verhinderte das Auftreten von neuen Vergiftungsfällen und begünstigte bei vergifteten Tieren den Verlauf.

- Therapeutisch wurden empfohlen: Entleeren des Magen-Darm-Trakts, antitoxische Mittel, Tonika, Kardiaka.

\section{Diskussion}

Die Ausführungen von Nicolau et al. lassen erkennen, dass die Vergiftung durch Gundelrebe im Zusammenhang mit Luzerne- fütterung durchaus dosisabhängig ist und selbst bei sehr hohen Anteilen von Gundelrebe in der Luzerne nicht alle Tiere erkranken.

Erstaunlich ist die Einmaligkeit des Ereignisses: Es erkrankten innerhalb weniger Tage in einem bestimmten Gebiet mehr als 40 Pferde. Von Vergiftungsfällen an anderen Orten zu anderen Zeiten ist seither nach Wissen der Autorinnen nicht berichtet worden. Dies lässt vermuten, dass weitere Faktoren nötig sind, damit es zu Vergiftungen in dem beschriebenen Ausmaß kommen kann (\$ Kasten 4). Unklar bleibt v.a., wie es überhaupt zu so hohen Anteilen an Gundelrebe in der Ration kommen kann.

\section{Ist eine Gundermann- Vergiftung in Deutschland grundsätzlich möglich?}

Hierzu einige ökologische Aspekte:

- Massenhaftes Vorkommen von Gundelrebe ist möglich.

- Als Mullbodenzeiger (Humusbildung aus Laub ebenso wie sich zersetzender, aufgedüngter Torf nach Entwässerung) liebt Gundelrebe humos-nährstoffreiche Böden, gerne kalkhaltig und bevorzugt gute Wasserversorgung. Daher wächst sie auch auf schweren Böden mit guter Feuchtigkeit. Nährstoffmangel und Trockenheit begrenzen das Wachstum und schwächen die Pflanze in der Konkurrenz mit anderen Pflanzen. Beschattung und Vertritt schaden der Konkurrenz oft mehr als der Gun- delrebe. Reichliche Phosphor-, vor allem aber Stickstoffgaben können zu besonders mastigen (d.h. großen und kräftigen), dunkelgrünen Pflanzen mit enormem Wachstum führen, die unter diesen günstigen Verhältnissen von voller Besonnung sogar profitieren. In Heuwiesen kann dann selbst Quecke von Gundelrebe überwachsen und bedeckt werden.

- In gut abgelagertem Heu ist Gundelrebe allerdings mit an Sicherheit grenzender Wahrscheinlichkeit ungiftig [8].

\section{Erzwungene „Weidepflege“ kann grundsätzlich zu Vergiftungen führen}

Säugetiere, also auch Pferde, wissen nicht instinktiv, welche Pflanze giftig ist. Sie „wissen“ aber, dass nicht alles fressbar ist. Daher werden neue Futtermittel zuerst sehr skeptisch in kleiner Menge getestet. Das Tier baut ein Geschmacks- und Geruchsgedächtnis für Futtermittel auf. Ist das neue Futtermittel gut bekömmlich, wird die Menge vorsichtig gesteigert. Das Pferd „weiß“, wann es welches Kraut oder welches Gras fressen möchte - und welche Geschmacks- und Geruchsstoffe es unbedingt meiden sollte.

Durch die Intensivierung der Landwirtschaft ist allerdings die Artenvielfalt extrem rückläufig, und die Pferde stehen ständig vor einem Zaun, der ihnen die gewünschten Futtermittel verwehrt. Schlimmer noch: Viele Pferdehalter zwingen ihre Pferde zum „Saubergrasen“. Bis die Weide nicht „wie ordentlich gemäht“ aussieht (bevor der Teller nicht absolut leer ist), gibt es nichts Neues: „So schlimm kann der Hunger nicht sein." Wer auf diese Weise Tiere zwingt, Pflanzen zu fressen, die ihnen nach ihrer Erfahrung nicht gut tun und die sie normalerweise meiden würden, trägt die Verantwortung für die resultierende Vergiftung.

\section{Die Chance zu meiden verhindert Vergiftung}

Sehr hungrige Tiere sind immer potenziell durch Vergiftungen gefährdet. Diese banale Tatsache gehört zu den wichtigsten Regelmechanismen zur Stabilisierung von natürlichen Weidesystemen: In den feuchten Klimazonen reguliert das Futterangebot die Populationsgröße der Herden. Dabei spielen Gifte in den Futterpflanzen eine bedeutende Rolle [15, 16]. Wer bei zu- 
nehmender Giftigkeit der Futterpflanzen nicht abwandert in weniger intensiv beweidete Landschaften, der muss mit Vergiftungen leben bzw. wird sterben, damit sich das Ökosystem erholen und sich langfristig eine stabile Lebensgrundlage für alle Lebewesen entwickeln kann. Alle schwachen und kranken (vergifteten) Tiere fallen dabei Beutegreifern zum Opfer.

\section{Fazit}

Bedenkt man, wie häufig Gundelrebe auf Pferdeweiden zu finden ist und dass seit 1955 keine Vergiftungen bekannt geworden sind, so scheint Gundelrebe als Giftpflanze für Pferde praktisch keine Rolle zu spielen.

Die bisher beschriebenen Intoxikationen wurden offenbar durch die besondere Fütterung bedingt: in allen Fällen alleinige Luzernefütterung mit sehr hohem Anteil (> 1/3 der Ration) Gundelrebe oder Gundelrebe als alleiniges Futtermittel.

Bei Betrachtung der Tatsachen erscheint die Aufregung den Fakten also nicht angemessen. Im Gegenteil: Die positiven Erfahrungen unserer Vorfahren und der modernen pharmakologischen Forschung mit der inhaltsstoffreichen Gundelrebe lassen aufhorchen und hoffen, dass die Gundelrebe in Zukunft eine ihr angemessene Stellung im Bewusstsein der Menschen erhält. @

\section{() Summary}

\section{Ground-ivy (Glechoma hederacea) and the cause of toxicosis in horses: In search of evidence}

Glechoma hederacea is suspected to be the cause of toxicosis in horses. The evidence from the literature leading to this suspicion shows the preconditions for the poisoning as: feeding the horse on impure lucerne (Medicago sativa) containing a high degree (>30\%) of ground ivy and alternatively, the experimental feeding of the horse exclusively on Glechoma hederacea.

\section{Key words}

Glechoma hederacea - Medicago sativa - toxicosis - horse

\section{( Literatur}

[1] Ahlswede L. Möglichkeiten der praktischen Pferdefütterung. In: Thein P et al. Handbuch Pferd. München: BLV; 2002: 154

[2] Barnes J, Anderson LA, Phillipson JD. Herbal Medicines. 2. Aufl. London: Pharmaceutical Press; 2002

[3] CliniTox. Management von Vergiftungen beim Pferd (2012). Im Internet: www.vetpharm.uzh. ch/clinitox/pfd/toxtpfd.htm; Stand: 17.06.2012

[4] Düll R, Kutzelnigg H. Taschenlexikon der Pflanzen Deutschlands. 6. Aufl. Wiebelsheim: Quelle \& Meyer; 2005

[5] Fröhner E. Lehrbuch der Arzneimittellehre für Thierärzte. 4. Aufl. Stuttgart: Enke; 1896

[6] Frohne D, Jensen U. Systematik des Pflanzenreichs. 5. Aufl. Stuttgart: Wissenschaftliche Verlagsgesellschaft; 1998

[7] Frohne D, Pfänder HJ. Giftpflanzen. Ein Handbuch für Apotheker, Ärzte, Toxikologen und Biologen. 4. Aufl. Stuttgart: Wissenschaftliche Verlagsgesellschaft; 1997

[8] von Hazslinszky B. Ueber Vergiftung bei Pferden durch Glechoma. Dtsch Tierärztl Wschr 1935; 43: 708-709

[9] Hiller K, Melzig MF. Lexikon der Arzneipflanzen und Drogen. 2. Aufl. Heidelberg: Spektrum Akademischer Verlag; 2010

[10] Kim J, Lee I, Ha D, Seo J, Min B, Yoo I, Bae K. New sesquiterpene lactones from Glechoma hederacea $\mathrm{L}$. and their cytotoxic effects on human cancer cell lines. Planta Med 2011; 77: 955-957

[11] Löscher W, Ungemach R, Kroker R, Hrsg. Pharmakotherapie bei Haus- und Nutztieren. 8. Aufl. Stuttgart: Enke in MVS Medizinverlage; 2010

[12] Nicolau A, Bârzã H, Duca H, Creteanu C, May H, Popoviciu A. Vergiftungen durch die Pflanze
Glechoma hederacea beim Pferde. Mh Vet Med 1956; 11: 534-538

[13] Pirkelmann H, Hrsg. Pferdehaltung. Stuttgart: Eugen Ulmer; 1991

[14] Roth L, Daunderer M, Kormann K. Giftpflanzen - Pflanzengifte. 5. Aufl. Hamburg: Nikol Verlagsgesellschaft; 2008

[15] Seldal T, Andersen KJ, Hogstedt G. Grazing-induced proteinase-inhibitors: a possible cause for lemming population cycles. Oikos 1994; 70: 3-11

[16] Vanselow R. Giftige Gräser auf Pferdeweiden. 3. Aufl. Hohenwarsleben: Westarp; 2011

[17] Wikipedia. Glechoma hederacea. Im Internet: http://en.wikipedia.org/wiki/Glechoma_hederacea; Stand: 4.6.2012

Online zu finden unter

http://dx.doi.org/10.1055/s-0032-1315014

\section{(- Dr. rer. nat. Renate Vanselow}

Sachverständige und ökologische Gutachterin BDBiol

Dorfstraße $97 \mathrm{~b}$

24232 Dobersdorf

E-Mail: renate.vanselow@t-online.de

Diplom-Biologin, Bereich Botanik, Fachgebiet Ökophysiologie, Promotion in der Ökosystemforschung, Mitarbeit in verschiedenen Projekten zum Klimawandel, danach angestellt an der Landwirtschaftskammer, seit 2002 freiberufliche Biologin, Schwerpunkt Beweidungssysteme. Autorin von Fachbüchern und Artikeln, Fachreferentin. Mitglied in der Berufsvertretung Deutscher Biologen.

- Dr. med. vet. Cäcilia Brendieck-Worm Talstraße 59

67700 Niederkirchen

E-Mail: caecilia@fworm.de

Geb. 1957, 1976-1981 Studium der Veterinärmedizin in Gießen, 1982-1986 Promotion am Institut für Tierzucht und Haustiergenetik der JLU Gießen, seit 1985 Mitglied einer tierärztlichen Praxis für Groß- und Kleintiere in der Pfalz, Zusatzbezeichnung und Weiterbildungsermächtigung Biologische Tiermedizin, Leitung des Arbeitskreises Phytotherapie der GGTM. 